\title{
Induction of dissociated cytokine profiles by ISRAA with selective critical involvement of ERK1/2 in its signaling functions
}

\author{
SAHAR ELHANNAN ${ }^{1}$, SAFA TAHA ${ }^{1}$, NOUREDDINE BEN KHALAF ${ }^{1}$, \\ HALLA BAKHEIT $^{1}$, M. DAHMANI FATHALLAH ${ }^{2}$ and MOIZ BAKHIET ${ }^{1}$ \\ ${ }^{1}$ Department of Molecular Medicine, Princess Al-Jawhara Center for Genetics and Inherited Diseases, College of Medicine \\ and Medical Sciences, ${ }^{2}$ Biotechnology Program, College of Graduate Studies, Arabian Gulf University, Manama, Bahrain
}

Received January 11, 2015; Accepted September 9, 2015

DOI: $10.3892 /$ ijmm.2015.2376

\begin{abstract}
The immune system-released activating agent (ISRAA) is an immune mediator activated as a result of a nerve stimulus initiated by immune challenge. We have previously demonstrated that ISRAA and tumor necrosis factor (TNF) receptor 1 (TNFR1) share an interspecies-conserved motif (72\% homology) that induces the apoptosis and proliferation of human peripheral blood mononuclear cells (hPBMCs) in a dose-dependent manner. In the present study, cytokine profiles were examined in response to the stimulation of hPBMCs with ISRAA. Furthermore, the signaling pathways induced by ISRAA were mapped. The results revealed high measurable levels of TNF- $\alpha$, interleukin (IL)-6, IL-8, IL-10 and interferon (IFN)- $\gamma$, but not IL-4, IL-17 (IL-17A) or transforming growth factor (TGF)- $\beta$. The analysis of signaling pathways revealed the activation of extracellular-regulated protein kinase (ERK)1/2 as a downstream signal in the mitogen-activated protein kinase (MAPK) pathway during TNF- $\alpha$ and IL-6 production and apoptosis, but not during proliferation following stimulation with ISRAA by triggering the Fas-associated protein with death domain (FADD). STAT3 was found to be unphosphorylated in the ISRAA-stimulated hPBMCs, and STAT3 was ubiquitously expressed in unstimulated cells, suggesting that ISRAA has a protein inhibitor of activated STAT (PIAS)-like activity, by functioning as a negative regulator of the effects of STAT3 on the Janus kinase (JAK)/STAT pathway. The determination of the nature of cytokine responses together with the signaling pathways of cellular activity induced by ISRAA paves the way for the investigation of a potential target of ISRAA and for the development of novel therapeutic approaches for the treatment of immune-regulated disorders.
\end{abstract}

Correspondence to: Professor Moiz Bakhiet, Department of Molecular Medicine, Princess Al-Jawhara Center for Genetics and Inherited Diseases, College of Medicine and Medical Sciences, Arabian Gulf University, P.O. Box 26671, Manama, Bahrain E-mail: moiz@agu.edu.bh

Key words: apoptosis, proliferation, tumor necrosis factor receptor 1, mitogen-activated protein kinase, Janus kinase/signal transducer and activator of transcription, Fas-associated protein with death domain

\section{Introduction}

The immune system-released activating agent (ISRAA) is a $15 \mathrm{kDa}$ novel immune mediator encoded by a gene located on mouse chromosome 14 (GenBank accession no. EU552928) (1) and is believed to mediate signaling between the nervous and the immune system. ISRAA was initially considered to have a cytokine-like activity due to several factors, including its relatively low molecular mass, its biological effects on a broad spectrum of cell types, such as $\mathrm{T}$ and $\mathrm{B}$ cells and monocytes/macrophages, the ability of the same cell populations to respond to ISRAA by producing cytokines, such as interferon (IFN)- $\gamma$, as well as the fact that ISRAA is lined up on chromosome 14 on the mouse system where several low molecular mass cytokines are mapped (1). Although ISRAA was identified in the mouse, the alignment has shown that the molecule has conserved regions between many species (2). Furthermore, the function of ISRAA has been found to be dose-dependent, as it has the ability to stimulate the proliferation of human peripheral blood mononuclear cells (hPBMCs) at a low concentration of $50 \mathrm{pg}$, while a higher concentration of $5 \mu \mathrm{g}$ has been shown to result in apoptosis (2).

In general, these two pathways for proliferation and apoptosis are controlled by a variety of (growth-deleted) factors that bind to receptors on the cell surface and initiate a cascade of events (signal transduction pathways), that convey the message from the receptors to the nucleus where transcription factors bind to DNA, turning either 'ON' or 'OFF' the production of proteins that induce cell proliferation or death (3-5). Such activities may be induced directly by the effective molecule, e.g., ISRAA, or indirectly by the production of immune mediators, such as cytokines (2). Cytokines are low molecular mass proteins $(\sim 5-20 \mathrm{kDa})$ that are important in cell signaling. They are released by cells and act on the same cell (autocrine) or on other cells in a paracrine or endocrine manner. Cytokines are produced by a broad range of cells, acting via receptors and are particularly important for the immune system as they modulate the balance between humoral and cell-mediated immune responses. In addition, they regulate the growth, maturation and responsiveness of particular cell populations. Thus, they are important in health and disease, and more specifically, in host responses to infection, immune responses, inflammation, trauma, sepsis, cancer and reproduction (6). 
The identification of ISRAA by our laboratory prompted us to investigate the mechanisms through which the activation of ISRAA initiates signaling cascades to induce particular effects, either directly or indirectly via cytokine production. We also wished to further characterize the signaling pathways involved in order to gain insight into the mechanisms through which ISRAA integrates the regulatory mechanisms and how information flows through a system.

Understanding signaling pathways is challenging, since there are hundreds of signaling pathways, as well as hundreds or even thousands of different proteins used by the cell at the same time. The fact that many signals are transient events further complicates our understanding of signaling pathways. Furthermore, the response of some pathways depends on the strength and the time of the stimulus $(7,8)$. In the present study, we stimulated hPBMCs with ISRAA in order to examine the broad spectrum of cytokines and explore the activated signaling pathways by detecting phosphorylated proteins from second messengers down to the nuclear translocation of transcription factors.

\section{Materials and methods}

Isolation of hPBMCs. This study was approved by the Ethics Committee of Arabian Gulf University, Manama, Bahrain. Blood samples in EDTA tubes (Becton, Dickinson and Co., San Diego, CA, USA) were obtained from apparently healthy donors; none of the participants reported any history of acute or chronic medical problems, and informed consent was obtained from all donors. hPBMCs were purified using the Ficoll-Hypaque technique by diluting the blood 1:2 with phosphate-buffered saline. Subsequently, $10 \mathrm{ml}$ of this diluted blood were carefully layered onto the $3 \mathrm{ml}$ Ficoll-Hypaque Plus cushion (Pharmacia Biotech, Uppsala, Sweden) and centrifuged at $400 \mathrm{x}$ g for 30-40 min at $18-20^{\circ} \mathrm{C}$. The upper layer was drawn off using a clean Pasteur pipette, leaving the lymphocyte layer undisturbed at the interface, which was then washed twice using phosphate-buffered saline and once with RPMI-1640 containing 1\% FBS. The pellet was reconstituted in $5 \mathrm{ml}$ of complete RPMI-1640 (Life Technologies, Grand Island, NY, USA) culture medium containing $10 \%$ heat-inactivated fetal bovine serum, $2 \mathrm{mM}$ L-glutamine, $10 \mathrm{mM}$ HEPES buffer and antibiotic $(50 \mathrm{U} / \mathrm{ml}$ penicillin, $50 \mu \mathrm{g} / \mathrm{ml}$ streptomycin). The viability of the mononuclear cells was confirmed by the trypan blue exclusion test. The viable cell numbers were counted using a hemocytometer. The viability of the mononuclear cells was $>95 \%$ on average, as previously demonstrated (9).

The obtained mononuclear cells were rapidly plated onto a tissue culture plate (Falcon 3001) at a concentration of $1 \times 10^{6}$ cells $/ \mathrm{ml}$ and incubated in a $37^{\circ} \mathrm{C}, 95 \%$ air, $5 \% \mathrm{CO}_{2}$, $100 \%$ humidity incubator. Subsequently, the cells were stimulated with $5 \mu \mathrm{g}$ and $50 \mathrm{pg}$ concentrations of ISRAA, and $5 \mu \mathrm{g}$ of phytohaemagglutinin (PHA; Cat. no. L8902; Sigma-Aldrich, St. Louis, MO, USA) as a positive control (PC). Cells without stimulation were used as a negative control (NC). Each concentration was used in triplicate. The cells were collected at $0,5,10$, 15 and $30 \mathrm{~min}$ and at 1,3, 6, 12,24 and $48 \mathrm{~h}$; however, stimulation for $24 \mathrm{~h}$ was found to be the optimal time point (data not shown).

Identification and production of ISRAA. ISRAA used in this study was obtained as previously described (1). The
QIAexpressionist ${ }^{\mathrm{TM}}$ protocol was used for the cloning of the full length ISRAA gene and for high-level expression and purification of the 6xHis-tagged protein according to the manufacturer's instructions (Qiagen, Inc., Valencia, CA, USA). In brief, protein expression and purification were performed by the transformation of two constructs of bacterial expression vector (pQE-32 vector) in $5 \mathrm{ml}$ Lysogeny broth (LB) culture medium; protein expression was induced using isopropyl $\beta-\mathrm{D}$ 1-thiogalactopyranoside (IPTG) for one construct, while the other construct was kept uninduced to serve as a negative control. The expression was tested on a small scale first, and was then tested on a large scale using $11 \mathrm{LB}$ culture medium. Protein purification was performed by immobilized-metal affinity chromatography (IMAC) as the initial purification step. In addition, column chromatography under denaturing conditions with Ni-NTA magnetic beads (Qiagen, Hilden, Germany) was carried out to achieve further purification. Subsequently, the protein was subjected to IMAC again to remove the protease and any cellular proteins that bound to the metal affinity resin, followed by sodium dodecyl sulphate polyacrylamide gel electrophoresis (SDS-PAGE) and mass spectrometry to determine the expression and purification of the ISRAA protein. Following elution, the purified protein showed 2.212 optical density (OD) using the $280 \mathrm{~nm}$ absorbance wavelength, and western blot analysis was carried out using anti-His antibody (Fig. 10) indicating a high level of protein purification, which was $80-95 \%$; polymyxin B was used to neutralize the effect of the Gram-negative capsule endotoxins. Purified recombinant protein when titrated showed that there are two concentrations considered to be optimal in inducing the proliferation and cytotoxicity of the mononuclear cells; these two concentrations were $50 \mathrm{pg}$ and $5 \mu \mathrm{g}$. To ensure that those two effects are due to the ISRAA recombinant protein, polyclonal antibodies to ISRAA were used, and the effect of ISRAA was neutralized by the antibodies.

MTT cell proliferation and cytotoxicity assay. The MTT Cell Proliferation Assay (ATCC, Manassas, VA, USA) was used to measure the cell proliferation rate. The protocol was performed to examine the proliferative and cytotoxic effects of ISRAA on the cells according to manufacturer's instructions. Cell suspension was harvested by centrifugation, and was then resuspended at $1 \times 10^{6}$ cells $/ \mathrm{ml}$. A total of $100 \mu \mathrm{l}$ of cell suspension was plated in each well in triplicate. Three control wells of medium were left alone to provide the blanks for the absorbance readings. Another three wells with cell suspension alone as a negative control and three wells stimulated with $5 \mu \mathrm{g}$ of PHA as a positive control were used. A total of $10 \mu \mathrm{l}$ of $50 \mathrm{pg}$ and $5 \mu \mathrm{g}$ ISRAA protein was added to each well in triplicate. The plates were incubated at $37^{\circ} \mathrm{C}$ in $5 \% \mathrm{CO}_{2}$ for $24 \mathrm{~h}$. Subsequently, $10 \mu \mathrm{l}$ of $5 \mathrm{mg} / \mathrm{ml}$ MTT reagent were added to each well, including controls and incubated for $4 \mathrm{~h}$ at $37^{\circ} \mathrm{C}$. A total of $100 \mu \mathrm{l}$ of MTT solvent (acidic isopropanol $0.04 \mathrm{M} \mathrm{HCl}$ in absolute isopropanol) was then added to all wells. The plates were covered with tinfoil and agitated on an orbital shaker for $15 \mathrm{~min}$. Finally, the absorbance at $690 \mathrm{~nm}$ was recorded, and the cytocide rate was calculated using the following formula: cytocide rate $(\%)=(\mathrm{OD}$ control - OD experiment)/OD control x100. The assay was also used to determine the effects of the mitogen-activated protein kinase (MAPK) pathway inhibitor, U0126 (MEK1/2 inhibitor, 
from Cell Signaling Technology, Inc, Danvers, MA, USA; Cat. no. 9903), on ISRAA-induced apoptosis. The inhibitors were added at a concentration of $10 \mu \mathrm{M} 1 \mathrm{~h}$ prior to stimulation.

Measurement of CD45/CD3/CD8/CD4-positive cell count by flow cytometry. To examine the effects of various concentrations of ISRAA on the absolute counts of mature T lymphocytes $\left(\mathrm{CD}^{+}\right)$and the percentage of helper/inducer $\left(\mathrm{CD}^{+} / \mathrm{CD}^{+}\right)$and suppressor/cytotoxic $\left(\mathrm{CD}^{+} / \mathrm{CD}^{+}\right) \mathrm{T}$ cell subsets, MultiTEST CD3 FITC/CD8, PE/CD45, PerCP/CD4 APC with Trucount tubes (Becton, Dickinson and Co.) containing a freeze-dried pellet of fluorescent beads in a single-use tube were utilized. Samples were stained directly in a Trucount tube. The lyophilized pellet in the tube was dissolved releasing a known number of fluorescent beads. During analysis, the absolute number (cells $/ \mu \mathrm{l}$ ) of positive cells in the sample was determined by comparing cellular events to bead events. A BD Biosciences flow cytometer was used for flow cytometry, as previously descrbied (10). The BD FACSCalibur ${ }^{\mathrm{TM}}$ flow cytometer was used with CaliBRITE ${ }^{\mathrm{TM}}$ beads and FACSComp version 4 and Multiset $^{\mathrm{TM}}$ Software as recommended by the manufacturer. This method was also used to determine the effects of the p42 MAPK pathway inhibitor (U0126) on ISRAA-induced proliferation. The inhibitors were added at a concentration of $10 \mu \mathrm{M} 1 \mathrm{~h}$ prior to stimulation.

Detection of cytokines by flow cytometry. To examine the effects of various concentrations of ISRAA at separate intervals on cytokine production BD Cytometric Bead Array (CBA), Human Soluble Protein and CBA Flex Set for the cytokines, interleukin (IL)-4, IL-6, IL-10, IL-17A, IFN- $\gamma$, tumor necrosis factor (TNF)- $\alpha$, IL- 8 and transforming growth factor (TGF)- $\beta$ (Becton, Dickinson and Co.) were used according to the manufacturer's instructions and as previously described (11). To determine the pathway adopted by ISRAA in the production of IL-6, pharmacological inhibitors of MAPKs [U0126, MEK1/2 inhibitor and SB20358, p38 inhibitor (Cell Signaling Technology, Inc; Cat. no. 5633)] were used and the levels of IL-6 were monitored using CBA with flow cytometry.

Quantitative sandwich enzyme-linked immunosorbent assay (ELISA) to detect IL-6. The hPBMCs were cultured and treated as described above. All the cells were incubated at $37^{\circ} \mathrm{C}$ in $5 \% \mathrm{CO}_{2}$ for $24 \mathrm{~h}$. The supernatants were collected and analyzed for IL-6 expression using an ELISA kit (R\&D Systems, Minneapolis, MN, USA).

Bradford protein assay. To perform the western blot analysis and immunofluorescence assay, the protein concentrations were measured to standardize the volume of protein extraction used on each reaction. The Bradford protein assay and nanogram measures were used (Sigma-Aldrich). This assay was performed in a 96 -well plate. A total of $5 \mu \mathrm{l}$ of a $0.1-1.4 \mathrm{mg} / \mathrm{ml}$ protein sample was used as a standard. The protein standards $(5 \mu \mathrm{l})$, the cell lysates and buffer as a blank were added to the 96-well plate. The reagent was gently mixed; subsequently, $250 \mu \mathrm{l}$ of the Bradford reagent were added and mixed in a shaker for $\sim 30 \mathrm{sec}$. The samples were incubated at room temperature for 5-45 min. The absorbance was then measured at $595 \mathrm{~nm}$. The protein concentration of the unknown samples was determined by comparing the Net A595 values against the standard curve, as previously described (12).

MAPK signaling pathway detection. MAPK activity was achieved by measuring the phosphorylation of extracellular signal-regulated protein kinase (ERK) $1 / 2$ pathways, as the main components of the MAPK downstream signaling protein which plays a vital role in the IL-6 signaling pathway. The hPBMCs were grown, counted and adjusted to $10^{6}$ cells $/ \mathrm{ml}$ in complete RPMI-1640 growth medium. The cells were treated with $5 \mu \mathrm{g}$ and $50 \mathrm{pg}$ concentrations of ISRAA in triplicate wells, and the cells were then collected at intervals of 5, 10, 30 and 60 min. Total cell extracts from Jurkat cells were treated with 12-O-tetradecanoylphorbol-13-acetate (TPA; Cell Signaling Technology, Inc) as a positive control, and the cell lysates were then tested for the level and the optimal time of phosphorylation by western blot analysis of the cell lysates for phosphorylated (p-)ERK1/2 [by incubating the membrane in p44/p42 MAPK (ERK/1/2 (Thr202/Tyr204; Cell Signaling Technology, Inc; Cat. no. 4370)]. The pharmacological inhibitor, U0126 (MEK1/2 inhibitor) (Cell Signaling Technology, Inc.), was used to examine the role of MAPK in the production of IL-6 as a direct or indirect effect of ISRAA. The cells were cultured (as described above), and then treated with $10 \mu \mathrm{M}$ of U0126 inhibitor for $60 \mathrm{~min}$ prior to stimulation with ISRAA at intervals of 5, 10 and $30 \mathrm{~min}$ and then examined by western blot analysis (see below) for p-ERK1/2 (13).

Signal transducer and activator of transcription (STAT)3 pathway detection. The hPBMCs were grown, counted and adjusted to $10^{6}$ cells $/ \mathrm{ml}$ in complete RPMI-1640 growth medium. The cells were treated with $50 \mathrm{pg}$ of ISRAA in triplicate wells. The cells were then collected after $0,5,10$, 30 and $60 \mathrm{~min}$. IFN- $\alpha$ treated Jurkat cells were used as a positive control and tested for the level and the optimal time of phosphorylation by western blot analysis of the cell lysates with p-Stat3 (Tyr705) antibody (Cell Signaling Technology, Inc.), as well as for the presence of unphosphorylated STAT3.

Western blot analysis. The cell lysates were subjected to electrophoresis on a $12 \%$ SDS-PAGE gel and transferred onto a nitrocellulose membrane. The membranes were blocked in Tris-buffered saline with 5\% milk and 0.1\% Tween-20. The blots were probed with anti-STAT3 (Cat. no. 9130), antip-Stat3 (Tyr705; Cat. no. 9130) and anti-p-p44/42 MAPK (ERK1/2) (Thr202/Tyr204; Cat. no. 4370) antibodies overnight and incubated with horseradish peroxidase-conjugated secondary antibody (Cat. no. 9130) (all from Cell Signaling Technology, Inc.). Antibody/antigen complex was visualized using an enhanced chemiluminescent ECL Western Blotting Substrate (Cell Signaling Technology, Inc.) scanned by an image analyzer (LAS-1000; Fujifilm, Tokyo, Japan), as previously described (13).

Immunostaining and fluorescence microscopy for ERK1/2. The hPBMCs were grown, counted and adjusted to $10^{6}$ cells $/ \mathrm{ml}$ in complete RPMI-1640 growth medium. The cells were treated with $50 \mathrm{pg}$ of ISRAA in triplicate wells. The cells were collected after $0,5,10,15,30$ and $60 \mathrm{~min}$, and were then fixed and stained directly in a multi-chamber slide. Using 


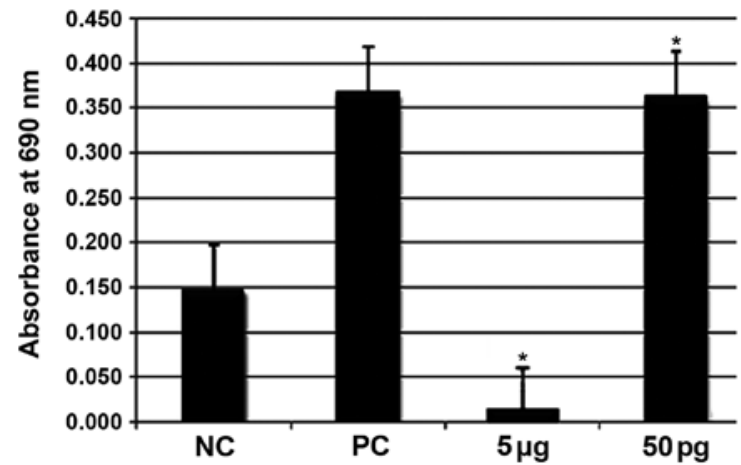

Figure 1. Effects of various concentrations of immune system-released activating agent (ISRAA) on cell proliferation and death. Human peripheral blood mononuclear cells (hPBMCs) were treated with $5 \mu \mathrm{g}$ and $50 \mathrm{pg}$ of ISRAA Phytohaemagglutinin (PHA) $(5 \mu \mathrm{g})$ was used as a positive control (PC) and unstimulated cells as a negative control (NC). Results were obtained by measuring the metabolic rate of cells as an estimate of the total number of cells involved in the reaction. Cells treated with $50 \mathrm{pg}$ ISRAA showed a significantly increased cell metabolic rate compared to cells treated with $5 \mu \mathrm{g}$ ISRAA, which resulted in significant cell death. Data are the means \pm SD; each value represents the mean of four experiments and each experiment was performed in triplicate. "p $<0.05$ vs. NC.

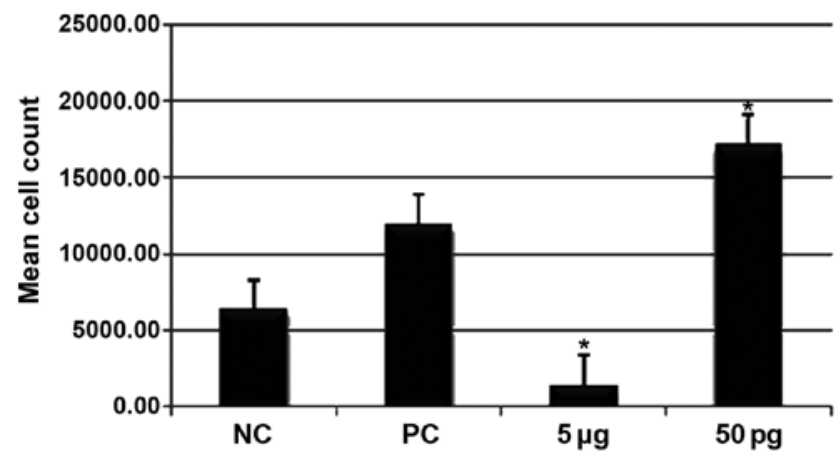

Figure 2. Effect of various concentrations of immune system-released activating agent (ISRAA) on human peripheral blood mononuclear cell (hPBMC) numbers. hPBMCs were treated with two different concentrations of ISRAA, i.e., $5 \mu \mathrm{g}$ and $50 \mathrm{pg}$. Phytohaemagglutinin (PHA) $(5 \mu \mathrm{g})$ was used as a positive control (PC) and unstimulated cells as a negative control (NC). Results were obtained by taking the absolute counts of leukocytes (CD45) and mature $\mathrm{T}$ lymphocytes $\left(\mathrm{CD}^{+}\right)$and the percentages of helper/inducer $\left(\mathrm{CD}^{+} / \mathrm{CD}^{+}\right)$ and suppressor/cytotoxic $\left(\mathrm{CD}^{+} / \mathrm{CD}^{+}\right) \mathrm{T}$ lymphocyte subsets were assessed by flow cytometry. Cells treated with $50 \mathrm{pg}$ of ISRAA showed significantly increased cell numbers compared to cells treated with $5 \mu \mathrm{g}$ of ISRAA, which showed a marked decrease in cell numbers. Data are the means $\pm \mathrm{SD}$; each value represents the mean of four experiments and each experiment was performed in triplicate. ${ }^{*} \mathrm{p}<0.05 \mathrm{vs}$. NC and treatment with $5 \mu \mathrm{g}$ ISRAA.

4\% formaldehyde in PBS, the cells were fixed for $15 \mathrm{~min}$ at room temperature, rinsed three times in PBS for $5 \mathrm{~min}$ each time and then permeabilized with $0.1 \%$ Triton X-100 and 5\% FCS in PBS at room temperature for $60 \mathrm{~min}$. The permeabilized cells were incubated with diluted primary antibody [p-p44/42 MAPK (ERK1/2) (Thr202/Tyr204) rabbit antibody; Cell Signaling Technology, Inc.] overnight at $4{ }^{\circ} \mathrm{C}$. They were subsequently washed three times in PBS for $5 \mathrm{~min}$ each time before the addition of the fluorochrome-conjugated secondary antibody [anti-rabbit, $\operatorname{IgG}(\mathrm{H}+\mathrm{L}), \mathrm{F}\left(\mathrm{ab} \mathrm{b}^{\prime}\right) 2$ fragment (Alexa Fluor ${ }^{\circledR} 488$ Conjugate); Cat. no. 4412; Cell Signaling Technology, Inc.], and were then kept for $1 \mathrm{~h}$ at room temperature in the dark and washed with PBS. Prior to visualization

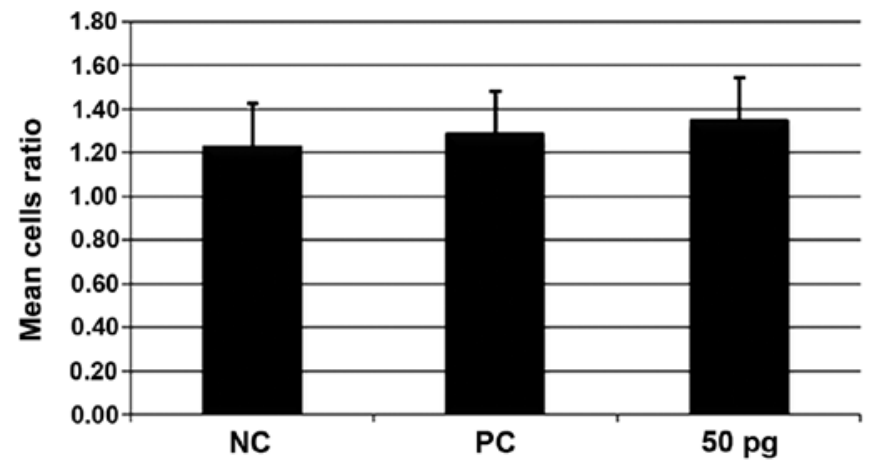

Figure 3. Effect of immune system-released activating agent (ISRAA) on the ratio of CD4:CD8 proliferated human peripheral blood mononuclear cells (hPBMCs). hPBMCs were stimulated with $50 \mathrm{pg}$ and $5 \mu \mathrm{g}$ of ISRAA for $24 \mathrm{~h}$. Phytohaemagglutinin (PHA) was used as a positive control (PC) and unstimulated cells as a negative control (NC). Cells were counted using the CD3/CD45/CD4/CD8 MultiTEST count by flow cytometry. The ratio of the numbers of the CD4 to the numbers of the CD8 was calculated using the software provided by the manufacturer, as detailed in the Materials and methods. Data are the means $\pm \mathrm{SD}$; each value represents the mean of four experiments and each experiment was performed in triplicate.

by fluorescence microscopy, the cells were covered with coverslip slides with ProLong ${ }^{\circledR}$ Gold Antifade Reagent (Cell Signaling Technology, Inc.; Cat. no. 9071). Images were obtained using a fluorescence microscope (Leica DM-3000; Leica Microsystems, Milton Keynes, UK), as previously described (13).

Statistical analysis. ANOVA was used to compare the levels of significance between the different groups. The Student's t-test was used to calculate the levels of significance. A value of $\mathrm{p}<0.05$ was considered to indicate a statistically significant difference.

\section{Results}

MTT cell proliferation and cytotoxicity assays. The effects of ISRAA on the cell metabolic rate were monitored by the MTT assay (Fig. 1). A significant decrease $(\mathrm{p}<0.05)$ in the number of cells was observed following treatment with the highest concentration of ISRAA $(5 \mu \mathrm{g})$. However, a significant increase in cell proliferation was observed following treatment with ISRAA at the concentration of $50 \mathrm{pg}(\mathrm{p}<0.05)$ compared to the unstimulated cells (negative control, NC). PHA was used as a positive control (PC) and was found to exert significant proliferative effects $(\mathrm{p}<0.05)$.

Effects of ISRAA on CD45/CD3/CD4/CD8-positive hPBMC numbers determined by flow cytometry. The cells treated with $50 \mathrm{pg}$ ISRAA exhibited a significant increase in the number of CD45/CD3/CD4/CD8-positive cells compared to the unstimulated cells and the cells treated with $5 \mu \mathrm{g}$ ISRAA ( $<0.05)$. Stimulation with $5 \mu \mathrm{g}$ ISRAA resulted in a significant decrease in the CD45/CD3/CD4/CD8-positive cell number $(\mathrm{p}<0.05)$ (Fig. 2).

Effect of ISRAA on the ratio of CD4:CD8 proliferated hPBMCs. The hPBMCs were stimulated for $24 \mathrm{~h}$ with $50 \mathrm{pg}$ 
A

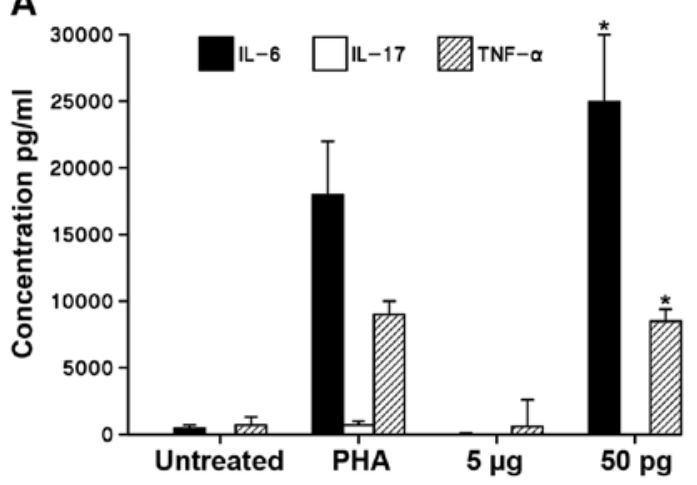

C

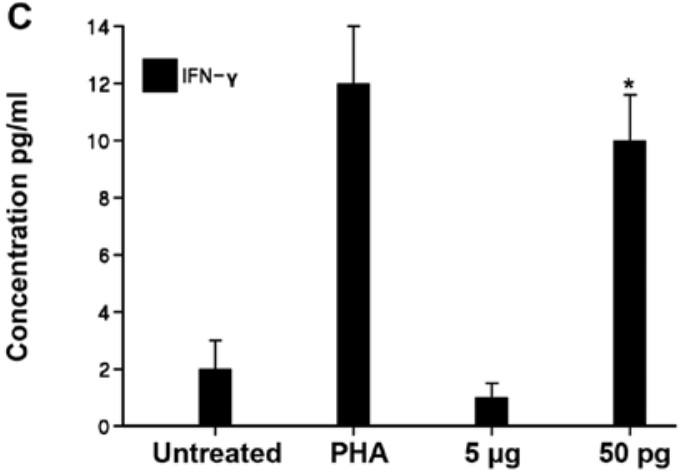

B
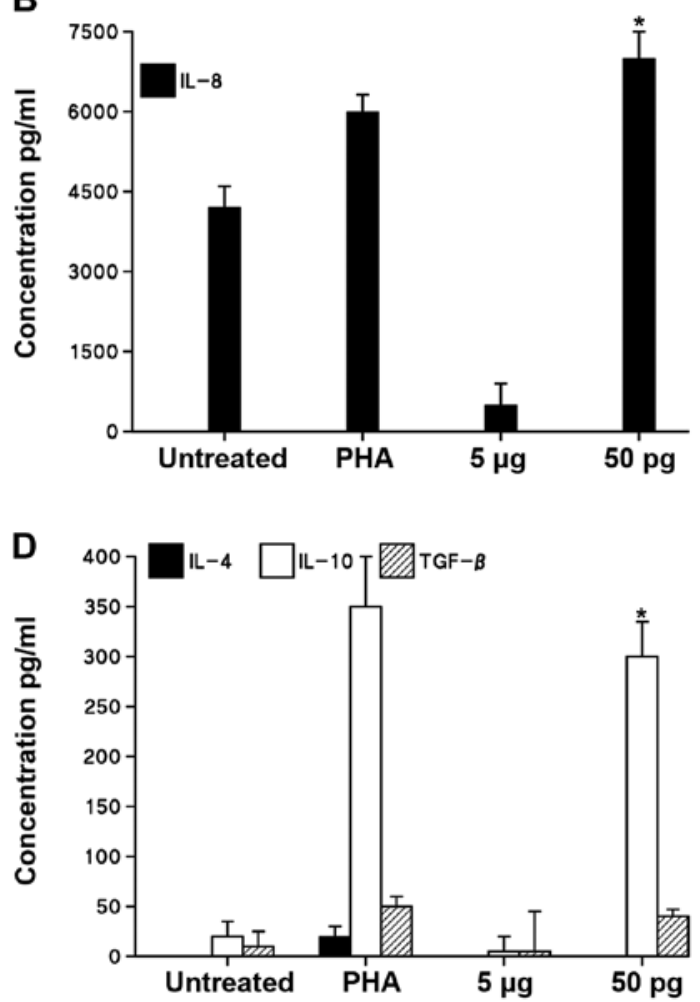

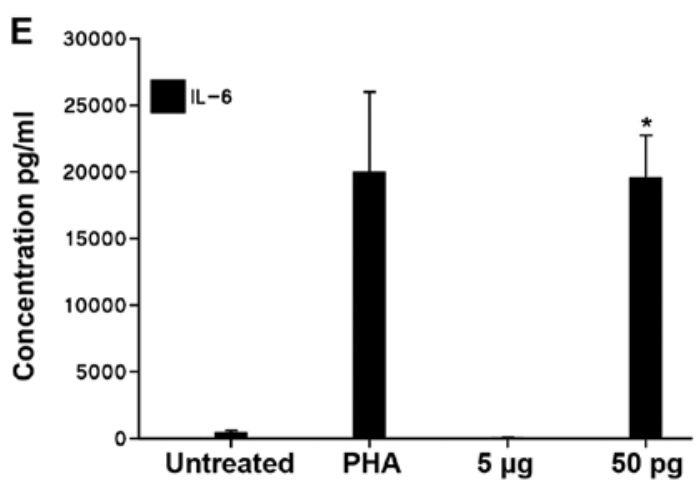

Figure 4. Cytokine induction as measured by Cytometric Bead Array (CBA) using flow cytometry and enzyme-linked immunosorbent assay (ELISA). Levels of cytokines (A) IL-6, IL-17, TNF- $\alpha$;, (B) IL-8, (C) IFN- $\gamma$ and (D) IL-4, IL-10, TGF- $\beta$ were measured after stimulating human peripheral blood mononuclear cells (hPBMCs) after being grown to (100 $\mu \mathrm{l}$ of $1 \times 10^{6}$ cells/well) with immune system-released activating agent (ISRAA) with concentrations of 50 pg and $5 \mu \mathrm{g}$ for $24 \mathrm{~h}$. The supernatant was collected and assayed for these cytokines using the CBA with flow cytometry; concentrations were expressed and compared with the basal level found on the unstimulated cells. Phytohaemagglutinin (PHA) was used as a positive control (PC) and unstimulated cells as a negative control (NC). Each value represents the mean of four experiments and each experiment was performed in triplicate. Error bars denote SD. (E) Similarly, the level of IL- 6 was measured by ELISA to confirm the above recorded level. ${ }^{*} \mathrm{p}<0.05$ vs. untreated cells (negative control).

and $5 \mu \mathrm{g}$ of ISRAA. PHA was used as a positive control (PC) and unstimulated cells served as a negative control (NC). The cells were counted using the CD45/CD3/CD4/CD8 MultiTEST count by flow cytometry. The cells stimulated with $50 \mathrm{pg}$ of ISRAA exhibited the ability to proliferate. In order to determine the subset of $\mathrm{T}$ lymphocytes which proliferated more as a result of stimulation with $50 \mathrm{pg}$ ISRAA, the ratio of the two subsets, i.e., the helper/inducer $\left(\mathrm{CD}^{+} / \mathrm{CD}^{+}\right)$and suppressor/cytotoxic $\left(\mathrm{CD} 3^{+} / \mathrm{CD}^{+}\right)$was calculated and the results are shown in Fig. 3. The T/helper and T/cytotoxic cells both increased in number at the same rate, with no significant difference observed between the groups (postive control and group treated with $50 \mathrm{pg}$ ISRAA; $>0.05$ ).
Cytokine production induced by ISRAA. To quantify and analyze the effects of ISRAA on the expression of a group of pro-inflammatory (IL-6, IL-17, IFN- $\gamma$, TNF- $\alpha$ ) and anti-inflammatory cytokines (IL- 8 , IL-4, IL-10, TGF- $\beta$ ) produced by the hPBMCs stimulated with $50 \mathrm{pg}$ and $5 \mu \mathrm{g}$ of ISRAA, various techniques were used, such as CBA with flow cytometry and quantitative sandwich ELISA. Kinetic analysis on cytokine production demonstrated that a 24-h incubation period is the optimal time point for the maximum induction of the measured cytokines (data not shown).

As shown in Fig. 4, with the use of CBA with flow cytometry, our results revealed that stimulation with $50 \mathrm{pg}$ ISRAA markedly increased the levels of the pro-inflammatory 


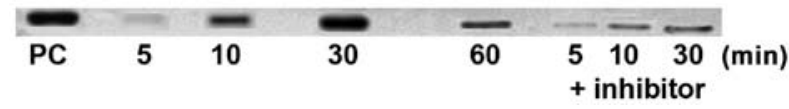

Figure 5. Western blot analysis of p-ERK1/2 with or without the addition of the inhibitor (U0126). Extracts from cells treated with $5 \mu \mathrm{g}$ and $50 \mathrm{pg}$ concentrations of immune system-released activating agent (ISRAA) with or without the ERK1/2 inhibitors at intervals of 5, 10,30 and $60 \mathrm{~min}$ (60 min were used only for cells stimulated with ISRAA with no inhibitor added). Total cell extracts from Jurkat cells treated with TPA (Cell Signaling Technology, Inc.) as a positive control (PC). Extracts were subjected to electrophoresis on a $12 \%$ SDS-PAGE ge and transferred onto a nitrocellulose membrane, probed with p-p44/42 MAPK (ERK1/2) overnight and incubated with horseradish peroxidase-conjugated secondary antibody. Antibody/antigen complex was visualized using an enhanced chemiluminescent ECL Western Blotting Substrate. The experiment was repeated several times with similar results. The figure represents ERK1/2 activation with the $50 \mathrm{pg}$ concentration of ISRAA.

cytokines, IL-6 and TNF- $\alpha(\mathrm{p}<0.05)$, but not those of IL-17 compared to the unstimulated cells (Fig. 4A). Stimulation with ISRAA $(50 \mathrm{pg})$ also induced a significant increase in the levels of IL-8 (Fig. 4B) and IFN- $\gamma$ (Fig. 4C) $(\mathrm{p}<0.05)$. The analysis of anti-inflammatory cytokines revealed a significant induction of IL-10 ( $\mathrm{p}<0.05)$, but not IL-4 or TGF- $\beta$ (Fig. 4D). The toxic concentration of $5 \mu \mathrm{g}$ ISRAA did not significantly alter the levels of all the examined cytokines.

The cytokine IL- 6 exhibited the highest detectable cytokine levels, as shown by CBA with flow cytometry. These results were confirmed by ELISA, which revealed a 4-fold increase in IL-6 production in the cells stimulated with $50 \mathrm{pg}$ ISRAA compared to the unstimulated cells $(\mathrm{p}<0.05$; Fig. 4E).

Western blot analysis of p-ERK1/2. The phosphorylation of ERK1/2 as a MAPK downstream signaling protein was examined by western blot analysis of extracts from cells treated with $50 \mathrm{pg}$ ISRAA with or without ERK1/2 inhibitor (U0126) at intervals of 5, 10,30 and $60 \mathrm{~min}$. Total cell extracts from Jurkat cells treated with TPA were used as a positive control. The results revealed that detectable ERK1/2 phosphorylation began at $10 \mathrm{~min}$ and steadily continued to increase until the 60 -min time point following stimulation, while low levels of phosphorylation or no phosphorylation were observed in cells stimulated with ISRAA when the inhibitors were used (Fig. 5).

ERK 1/2 translocation. Upon activation, ERK1/2 translocates to the nucleus in order to be transcribed. Using immunofluorescence assay to reveal the exact time required for $\mathrm{p}-\mathrm{ERK} 1 / 2$ to translocate to the nucleus, the results demonstrated that the phosphorylation and dimerization of ERK1/2 began at around $5 \mathrm{~min}$, and intensified at around $10 \mathrm{~min}$. It then began to translocate from the cytoplasm towards the nucleus at $15 \mathrm{~min}$ where some of the phosphorylated molecules entered the nucleus. This intensified at $30 \mathrm{~min}$, and by $60 \mathrm{~min}$, a complete translocation to the nucleus had occurred (Fig. 6).

Effect of ERK1/2 inhibitor on cell proliferation and apoptosis. The hPBMCs were cultured and treated with $5 \mu \mathrm{g}$ or $50 \mathrm{pg}$ of ISRAA with or without the p-ERK1/2 inhibitor. Data were obtained using the MTT assay for cell death (Fig. 7A), and cell proliferation was analyzed by taking the absolute counts of mature helper/inducer $\left(\mathrm{CD}^{+} / \mathrm{CD}^{+}\right)$and suppressor/cytotoxic $\left(\mathrm{CD}^{+} / \mathrm{CD}^{+}\right) \mathrm{T}$ lymphocyte subsets by flow cytometry (Fig. 7B). As shown in Fig. 7, the results revealed that, compared to the unstimulated cells (negative control, $\mathrm{NC}$ ), there was a significant increase in hPBMC proliferation $(\mathrm{p}<0.05)$ following stimulation with $50 \mathrm{pg}$ of ISRAA with or without the addition of the inhibitor. However, the toxic effect of the $5 \mu \mathrm{g}$ concentration of ISRAA was significantly abrogated by the addition of the inhibitor and, consequently, a significant increase in proliferation was observed following the addition of the inhibitor $(\mathrm{p}<0.05)$. PHA was used as a positive control $(\mathrm{PC})$ and did not significantly affect proliferation $(\mathrm{p}<0.05)$.

Effects of ERK1/2 inhibitors on the production of IL-6. To determine the pathway adopted by ISRAA in the production of IL-6, the pharmacological inhibitors of MAPKs,
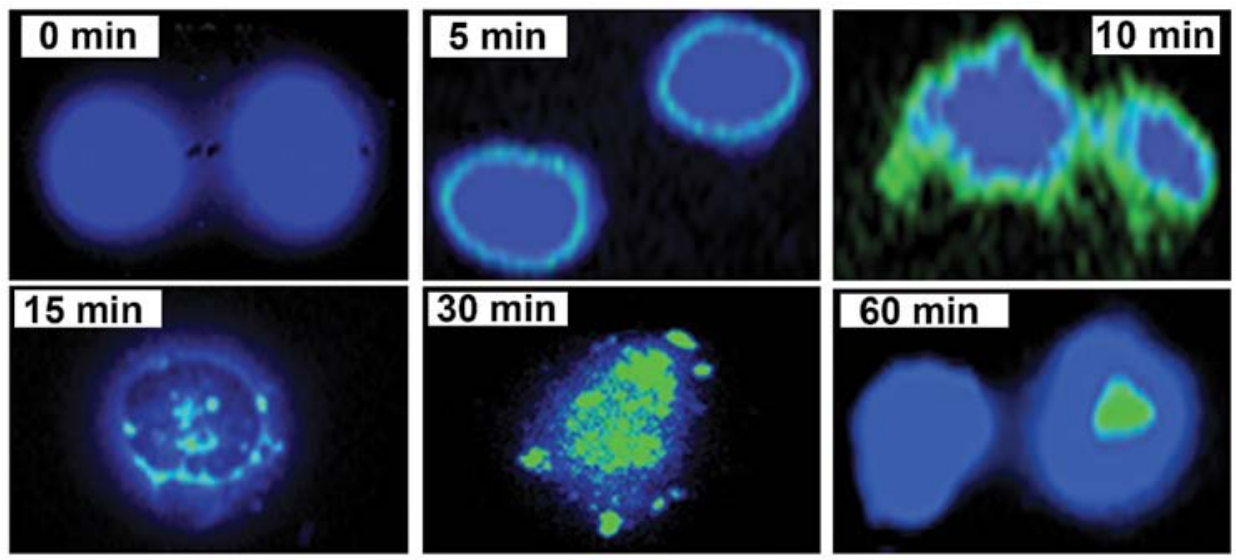

Figure 6. Immunofluorescenc assay for the p-ERK1/2 nuclear translocation. Cells were prepared as described in the Materials and methods, adjusted to $10^{6}$ cells/ $\mathrm{ml}$ and treated with $50 \mathrm{pg}$ of immune system-released activating agent (ISRAA). Cells were then collected at intervals of $0,5,10,15,30$ and 60 min. Thereafter, they were fixed and stained directly in multi-chamber slides using $4 \%$ formaldehyde in PBS for 15 min at room temperature, permeabilized for 60 min and incubated with primary antibody (ERK1/2) overnight at $4^{\circ} \mathrm{C}$. Subsequently, the fluorochrome-conjugated secondary antibody was added and kept for $1 \mathrm{~h}$ at room temperature in the dark, visualized by darkfield microscopy at x40 magnification. Accuracy was confirmed at a higher magnification (x100 magnification). Each test was performed in duplicate. 

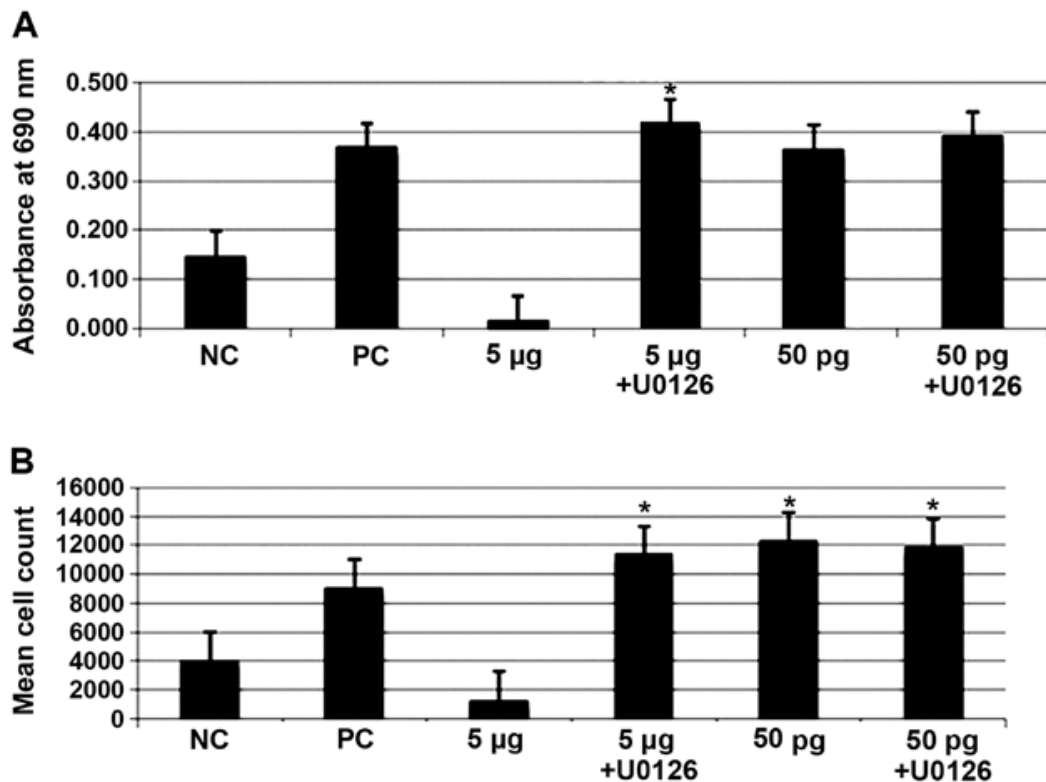

Figure 7. Effect of ERK1/2 inhibitor (U0126) on cell proliferation and death. Human peripheral blood mononuclear cells (hPBMCs) were treated with $5 \mu \mathrm{g}$ and $50 \mathrm{pg}$ of immune system-released activating agent (ISRAA) with or without the addition of $10 \mu \mathrm{M}$ of U0126, the ERK1/2 inhibitor which was added $1 \mathrm{~h}$ prior to stimulation. (A) MTT assay was used to measure cell death and (B) CD45/CD3/CD4/CD8 with flow cytometry was used to measure cell proliferation. Data are the means $\pm \mathrm{SD}$; all the experiments were repeated several times with similar results. ${ }^{*} \mathrm{p}<0.05$ vs. NC.

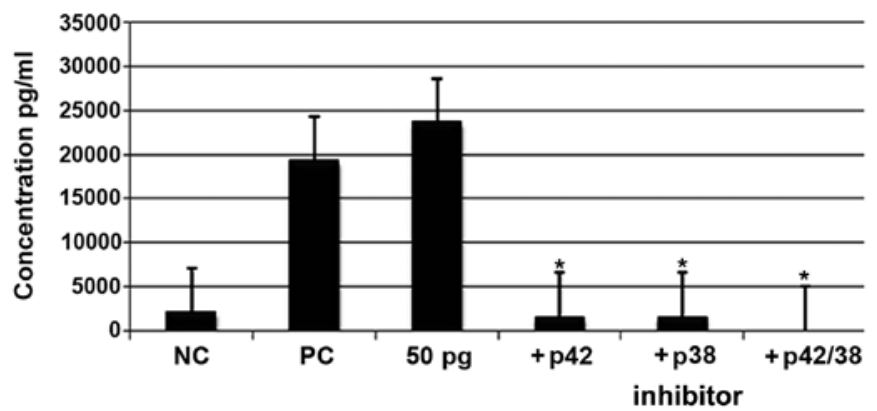

Figure 8. Inhibition of IL-6 production by the specific ERK1/2 (p42/38) inhibitors (p42, U0126; p38, SB203580). Human peripheral blood mononuclear cells (hPBMCs) from apparently healthy donors $\left(100 \mu 1\right.$ of $1 \times 10^{6}$ cells/well) after being grown to confluency pre-treated with U0126 and SB203580 (10 $\left.\mu \mathrm{M}\right)$ for $1 \mathrm{~h}$ prior stimulation with $50 \mathrm{pg}$ immune system-released activating agent (ISRAA) for $24 \mathrm{~h}$. The supernatant was collected and assayed for IL-6 by flow cytometry [Cytometric Bead Array (CBA)] assay. Concentrations were expressed and compared with the basal level of IL-6 on the unstimulated cells [negative control (NC)] and the positively stimulated phytohaemagglutinin (PHA) cells [positive control (PC)]. Each value represents the mean of at least four experiments, each performed in duplicate. Error bars denote SD. "p $<0.05$ vs. NC.

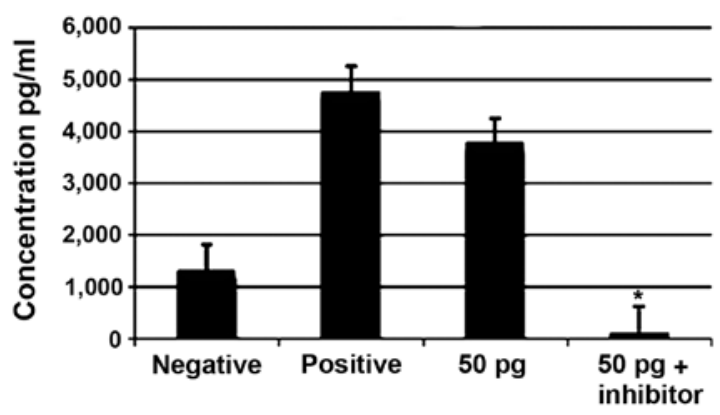

Figure 9. Effect of ERK1/2 inhibitor (U0126) on the production of TNF- $\alpha$. Human peripheral blood mononuclear cells (hPBMCs) were prepared as described above and pre-treated with $\mathrm{U} 0126(10 \mu \mathrm{M})$ for $1 \mathrm{~h}$ prior to stimulation with $50 \mathrm{pg}$ immune system-released activating agent (ISRAA) for $24 \mathrm{~h}$ The supernatant was collected and assayed for TNF- $\alpha$ by flow cytometry. Concentrations were expressed and compared with the basal level of TNF- $\alpha$ on the unstimulated cells, [negative control ( $\mathrm{NC})$ ] and the positively stimulated phytohaemagglutinin (PHA) cells [positive control (PC)]. Each value represents the mean of at least four experiments and each was performed in duplicate. Error bars denote SD. " p $<0.05$ vs. treatment with $50 \mathrm{pg}$ ISRAA.
U0126 and SB20358, were used and the levels of IL-6 were monitored using CBA with flow cytometry. Our results demonstrated the abrogation of IL-6 production as a result of pre-incubation with the two blockers prior to stimulation with ISRAA ( $<<0.05$; Fig 8$)$.

Effect of ERK1/2 inhibitors on the production of TNF- $\alpha$. Treatment with $50 \mathrm{pg}$ of ISRAA with or without the addition of the ERK1/2 inhibitor using the flow cytometric technique revealed a significant decrease in the expression of TNF- $\alpha$ when using the inhibitor compared with the increased level observed in the cells treated with $50 \mathrm{pg}$ of ISRAA ( $<<0.05$; Fig 9).

Phosphorylation of STAT3 proteins. IL-6 was originally cloned as an acute-phase response factor resulting in the activation of the Janus kinase (JAK)/STAT3 pathway. To examine the phosphorylation of the STAT3, western blot 


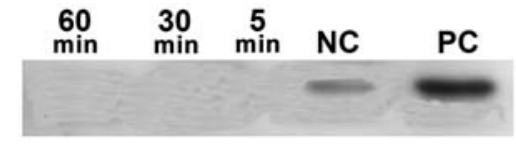

Figure 10. Phosphorylation of signal transducer and activator of transcription (STAT)3 proteins. Human peripheral blood mononuclear cells (hPBMCs) from apparently healthy donors ( $100 \mu 1$ of $1 \times 10^{6}$ cells/well) were stimulated with $50 \mathrm{pg}$ immune system-released activating agent (ISRAA) at intervals of 5,30 , and $60 \mathrm{~min}$. The cell extracts were subjected to western blot analysis. The blots were probed with unphosphorylated signal transducer and STAT3 antibody overnight and incubated with horseradish peroxidase-conjugated secondary antibody. Antibody/antigen complex was visualized using an enhanced chemiluminesence (ECL) Western Blotting Substrate. p-STAT3 protein was used as a positive control (PC).

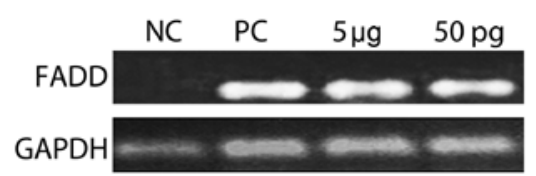

Figure 11. Fas-associated protein with death domain (FADD) expression. Human peripheral blood mononuclear cells (hPBMCs) (100 $\mu 1$ of $1 \times 10^{6}$ cells/well) were treated with immune system-released activating agent (ISRAA) at a high-dose concentration of $5 \mu \mathrm{g}$ and a low-dose concentration of $50 \mathrm{pg}$. Unstimulated cells were used as a negative control (NC), while cells treated with phytohaemagglutinin (PHA) at a concentration of $5 \mu \mathrm{g}$ $\mathrm{ml}$ were used as a positive control (PC). GAPDH was used as endogenous internal control. Target mRNA of FADD is shown as documented on the electrophoresis gel.

analysis was performed using monoclonal antibodies to phosphorylated and unphosphorylated STAT3. The results revealed that STAT3 factors were ubiquitously expressed in an unstimulated inactive unphosphorylated form. The examination of p-STAT3 revealed no phosphorylation of STAT3 (Fig. 10).

Fas-associated protein with death domain (FADD) expression. The expression of the death domain revealed that ISRAA activated FADD at a concentration of $5 \mu \mathrm{g}$ and $50 \mathrm{pg}$ (Fig. 11).

\section{Discussion}

ISRAA is an immune mediator activated upon a nerve stimulus triggered by an immune challenge (1). The human counterpart of mouse ISRAA is not known; however, we recently demonstrated that ISRAA contains an interspecies-conserved functional motif sharing $72 \%$ homology with a motif present in the TNF receptor 1 (TNFR1), a receptor connected to intracellular domains inducing dose-dependent signals for survival or death, and the potential proliferative effects of mouse ISRAA on human cells were recorded (2). In this study, we reconfirmed this activity using different techniques and examined the induction of a broad spectrum of pro-inflammatory and anti-inflammatory cytokines in response to the stimulation of hPBMCs with ISRAA. Furthermore, the signaling pathways used by ISRAA to induce cytokine production were explored.

The results revealed increased levels of IL-6, IL-8, IL-10, IFN- $\gamma$ and TNF- $\alpha$ produced by the ISRAA-stimulated hPBMCs, whereas no changes were observed in the production of the IL-4, IL-17A and TGF- $\beta$ cytokines in response to ISRAA stimulation. Since ISRAA is involved in different cellular activities, including death, growth and survival, complex signaling pathways may be involved. Moreover, dissociated cytokine profiles were recorded to induce such activities considering that cytokines are signaling proteins with pleiotropic effects in diverse aspects of bodily functions in health and disease and are used extensively in intercellular communications to produce different activities. Among the produced cytokines, IL- 6 showed the highest measurable levels in the current study.

IL-6 possesses a number of functional properties and this is reflected by the terminology used to describe the activities of this cytokine (IFN- $\beta 2$, hepatocyte-stimulating factor, cytotoxic $\mathrm{T}$ cell differentiation factor, $\mathrm{B}$ cell differentiation factor, and B cell stimulatory factor 2) (14). It is well known for promoting inflammatory events through the expansion and activation of $\mathrm{T}$ cells, the differentiation of $\mathrm{B}$ cells, and the induction of acute-phase reactants cells. By contrast, it also plays a protective role during disease and counteracts the manifestation of certain inflammatory responses (15-17). Thus, IL-6 functions not only as a pro- but also as an anti-inflammatory cytokine, and, as a result, plays a pivotal role during disease.

To understand the mechanisms through which ISRAA transduces its potent biological responses, we briefly examined the signaling pathway used by ISRAA to induce the production of IL-6. The signaling pathways known to be involved in the production of IL- 6 have been mainly found to be the MAPK and/or JAK/STAT pathways (18). Therefore, these two pathways were analyzed after stimulating the hPBMCs cells with ISRAA.

The ERK-MAPK pathway was the first signal transduction cascade to be unraveled and delineated from the cell membrane to the nucleus (4). The pathway has been extensively investigated and is also known as the classical mitogen kinase cascade $(19-23,28)$. The cascade is initiated by the small $\mathrm{G}$ protein Ras, which recruits the MAPKKKs A-Raf, B-Raf and Raf-1, the MAPKKs MEK1 and 2, and the MAPKs ERKs, in addition to a large number of a range of substrates, including membrane and cytoskeletal proteins, cytosolic enzymes, and transcription factors (24). Ras/MAPK regulates many cellular processes and plays an important role in physiological processes, such as proliferation, differentiation and development $(25,26)$. Moreover, Ras/MAPK has also been found to control the signaling pathway leading to the phosphorylation and inactivation of a pro-apoptotic Bcl-2 family member in mammalian cells, Bad, which is targeted on serine 112 by a MEK-dependent pathway, whereas the phosphorylation of two other residues occurs in a MEK-independent manner (one of them is serine 136 by the PI3K/AKT pathway). Serine 112 phosphorylation has been found to be required for the dissociation of Bad from Bcl-2 providing a key link between growth and survival pathways and protection from apoptosis (27).

ERK1/2 signaling has been implicated as a key regulator of cell proliferation, and for this reason, inhibitors of the ERK pathway are used in clinical trials as potential anticancer agents (28). In this study, we examined the involvement of ERK1/2 and p38 kinase in mediating IL-6 production from hPBMCs using U0126 and SB203580 as inhibitors of ERK1/2 and p38 kinase, respectively. We demonstrated that by treating the cells with U0126 and SB203580 for $1 \mathrm{~h}$ prior to stimulation 
with ISRAA, the differential effects of these compounds were observed in the level of IL-6, which was markedly decreased, suggesting the essential role and function of MAPKs in the level of IL-6 produced by cells stimulated with ISRAA.

In the present study, western blot analysis and immunofluorescence assay were used to investigate the levels and intensity of p-ERK (Thr202/Tyr204) as a downstream target protein, and the results revealed that P-ERK1/2 p-ERK1/2 as downstream signals in the MAPK pathway were phosphorylated due to ISRAA stimulation and a significant amount of p-ERK1/2 accumulated in the nucleus. The localization of MAPK depends on its phosphorylation state at the sites of action rather than on its activity. Phosphorylation induces its cyto-dimerization, which is required for its nuclear translocation. Upon activation, both kinases partly translocate to the nucleus $(29,30)$.

IL-6 signals via gp130 homodimerization which then activates JAKs and recruits STAT proteins (31). STAT factors are ubiquitously expressed in an unstimulated, inactive (unphosphorylated) form (32), predominantly regulated by post-transitional modifications, i.e., tyrosine and serine phosphorylation. This is in accordance with our findings since unphosphorylated STAT3 in the ISRAA-stimulated hPBMCs was noted, and STAT3 was ubiquitously expressed in unstimulated cells suggesting that ISRAA has a protein inhibitor of activated STAT (PIAS)-like activity by acting as a negative regulator of the JAK/STAT pathway, where PIAS1 and PIAS3 have been shown to inhibit the activity of STAT1 and STAT3, respectively (33).

The effects of ISRAA on cells have been shown to be dualistic, as high concentrations induce apoptosis and low concentrations induce proliferation (2). This study demonstrated that ERK1/2 had no effect on the ISRAA-induced cell proliferation, while it was shown that ERK1/2 is required for ISRAA-induced apoptosis since the inhibition of ERK activities by a small molecule inhibitor almost completely inhibited the apoptotic effects of the high dose of ISRAA and reversed the suppressive effect of the cytotoxic dose. This is in agreement with the results of other studies (34-36), suggesting that the ERK pathway mediates the apoptosis induced by different stimuli in different tissues even though ERK has generally been considered a survival signaling pathway. However, the mechanisms through which ERK mediates apoptosis remain unclear. We hypothesized that the ERK1/2 pathway is involved in apoptosis by increasing an upstream signal for TNF- $\alpha$ production via the extrinsic pathway by activating the death domain of the FADD pathway. This is supported by Jo et al (37), who examined the effect of ERK inhibition on TNF- $\alpha$ expression and subsequent caspase- 3 activation in cisplatin-induced acute renal failure in mice. The authors demonstrated that the inhibition of ERK1/2 reduced TNF- $\alpha$ expression, caspase- 3 activation and apoptosis in kidney tissue, suggesting that the ERK1/2 pathway also participates in apoptosis by increasing an upstream signal for TNF- $\alpha$ production. In this study, we also demonstrated that the TNF- $\alpha$ level induced by ISRAA was significantly decreased when ERK1/2 was inhibited, indicating that ISRAA/ERK1/2 may use the extrinsic apoptotic pathway by activating the death receptors via increasing the level of TNF- $\alpha$ to induce apoptosis. These data have been recently supported by an exhaustive structural analysis of ISRAA that demonstrated features of novel signaling molecules (unpublished data).
In conclusion, the present study confirmed the dose-dependent apoptotic and proliferative effects of ISRAA on hPBMCs. Furthermore, high measurable levels of dissociated cytokine responses were detected, and the downstream signals of the MAPK pathway (ERK1/2) were found to be critically involved. The results also revealed that ISRAA functions as a PIAS-like protein, functioning as a negative regulator of STAT3 action on the JAK/STAT pathway. Since FADD was activated with both concentrations of ISRAA (proliferative and toxic) and ERK1/2 activation was involved in the IL- 6 and TNF- $\alpha$ production and in apoptosis, but not in proliferation, further research into the intrinsic pathways is required to examine the effects of ISRAA on ERK and consequently on different caspases, and p53 and Bcl-2 proteins, as well as on survival signaling, such as the Ras/PI3K/Akt pathway and Fyn expression. Experiments to define the proliferative and apoptotic concentrations for each hPBMC population during proliferation and apoptosis have been recently initiated in our laboratory.

\section{Acknowledgements}

This study received financial support from the College of Medicine and Medical Sciences, Arabian Gulf University (Bahrain).

\section{References}

1. Bakhiet $\mathrm{M}$ and Taha S: A novel nervous system-induced factor inducing immune responses in the spleen. Immunol Cell Biol 86: 688-699, 2008

2. Taha S, Fathallah MD and Bakhiet M: An interspecies conserved motif of the mouse immune system-released activating agent (ISRAA) induces proliferative effects on human cells. Mol Med Rep 10: 75-81, 2014.

3. Arch RH, Gedrich RW and Thompson CB: Tumor necrosis factor receptor-associated factors (TRAFs) - a family of adapter proteins that regulates life and death. Genes Dev 12: 2821-2830, 1998

4. Chang F, Steelman LS, Shelton JG, Lee JT, Navolanic PM, Blalock WL, Franklin R and McCubrey JA:Regulation of cell cycle progression and apoptosis by the Ras/Raf/MEK/ERK pathway (Review). Int J Oncol 22: 469-480, 2003.

5. Elmore S: Apoptosis: a review of programmed cell death. Toxicol Pathol 35: 495-516, 2007.

6. Ibelgaufts H: Cytokines. In: Cytokines \& Cells Online Pathfinder Encyclopedia. Version 31.4. Spring/Summer 2013 edition.

7. Pollard TD, Earnshaw WC, Lippincott-Schwartz J and Johnson GT: Cell biology. 2nd edition. Saunders/Elsevier, Philadelphia, 2008.

8. Ubersax JA and Ferrell JE Jr: Mechanisms of specificity in protein phosphorylation. Nat Rev Mol Cell Biol 8: 530-541, 2007.

9. Sallusto F and Lanzavecchia A: Efficient presentation of soluble antigen by cultured human dendritic cells is maintained by granulocyte/macrophage colony-stimulating factor plus interleukin 4 and downregulated by tumor necrosis factor alpha. J Exp Med 179: 1109-1118, 1994.

10. Cairo MS, Wagner EL, Fraser J, Cohen G, van de Ven C, Carter SL, Kernan NA and Kurtzberg J: Characterization of banked umbilical cord blood hematopoietic progenitor cells and lymphocyte subsets and correlation with ethnicity, birth weight, sex, and type of delivery: A Cord Blood Transplantation (COBLT) Study report. Transfusion 45: 856-866, 2005.

11. Morgan E, Varro R, Sepulveda H, et al: Cytometric bead array: A multiplexed assay platform with applications in various areas of biology. Clin Immunol 110: 252-266, 2004.

12. Bradford MM: A rapid and sensitive method for the quantitation of microgram quantities of protein utilizing the principle of protein-dye binding. Anal Biochem 72: 248-254, 1976.

13. Leonard M, Ryan MP, Watson AJ, Schramek H and Healy E: Role of MAP kinase pathways in mediating IL-6 production in human primary mesangial and proximal tubular cells. Kidney Int 56: 1366-1377, 1999. 
14. Jones SA, Horiuchi S, Topley N, Yamamoto N and Fuller GM: The soluble interleukin 6 receptor: Mechanisms of production and implications in disease. FASEB J 15: 43-58, 2001.

15. Rao MS, Sun Y, Escary JL, Perreau J, Tresser S, Patterson PH, Zigmond RE, Brulet P and Landis SC: Leukemia inhibitory factor mediates an injury response but not a target-directed developmental transmitter switch in sympathetic neurons. Neuron 11: 1175-1185, 1993.

16. Yamamori T, Fukada K, Aebersold R, Korsching S, Fann MJ and Patterson PH: The cholinergic neuronal differentiation factor from heart cells is identical to leukemia inhibitory factor Science 246: 1412-1416, 1989.

17. Lo DC: A central role for ciliary neurotrophic factor? Proc Natl Acad Sci USA 90: 2557-2558, 1993.

18. Heinrich PC, Behrmann I, Haan S, Hermanns HM, Müller-Newen G and Schaper F: Principles of interleukin (IL)-6-type cytokine signalling and its regulation. Biochem J 374: $1-20,2003$.

19. Galabova-Kovacs G, Kolbus A, Matzen D, Meissl K, Piazzolla D Rubiolo C, Steinitz K and Baccarini M: ERK and beyond: insights from B-Raf and Raf-1 conditional knockouts. Cell Cycle 5: 1514-1518, 2006.

20. Kolch W: Meaningful relationships: the regulation of the Ras/ Raf/MEK/ERK pathway by protein interactions. Biochem J 351 Pt 2: 289-305, 2000.

21. Pagès G, Guérin S, Grall D, Bonino F, Smith A, Anjuere F, Auberger $P$ and Pouysségur J: Defective thymocyte maturation in p44 MAP kinase (Erk 1) knockout mice. Science 286: 1374-1377, 1999.

22. Pawson T: Regulation and targets of receptor tyrosine kinases Eur J Cancer 38 (Suppl 5): S3-S10, 2002.

23. Rouse J, Cohen P, Trigon S, Morange M, Alonso-Llamazares A, Zamanillo D, Hunt T, Nebreda AR: A novel kinase cascade triggered by stress and heat shock that stimulates MAPKAP kinase-2 and phosphorylation of the small heat shock proteins. Cell 78: 1027-1037, 1994

24. Galabova-Kovacs G, Catalanotti F, Matzen D, Reyes GX Zezula J, Herbst R, Silva A, Walter I and Baccarini M: Essential role of $\mathrm{B}$-Raf in oligodendrocyte maturation and myelination during postnatal central nervous system development. J Cell Biol 180: 947-955, 2008.

25. Shaul YD and Seger R: The MEK/ERK cascade: From signaling specificity to diverse functions. Biochim Biophys Acta 1773: 1213-1226, 2007.
26. Yoon S and Seger R: The extracellular signal-regulated kinase: Multiple substrates regulate diverse cellular functions. Growth Factors 24: 21-44, 2006.

27. Kyriakis JM and Avruch J: Mammalian mitogen-activated protein kinase signal transduction pathways activated by stress and inflammation. Physiol Rev 81: 807-869, 2001.

28. Roux PP and Blenis J: ERK and p38 MAPK-activated protein kinases: A family of protein kinases with diverse biological functions. Microbiol Mol Biol Rev 68: 320-344, 2004.

29. Brunet A, Roux D, Lenormand P, Dowd S, Keyse S and Pouysségur J: Nuclear translocation of p42/p44 mitogen-activated protein kinase is required for growth factor-induced gene expression and cell cycle entry. EMBO J 18: 664-674, 1999.

30. Lenormand P, Sardet C, Pagès G, L'Allemain G, Brunet A and Pouysségur J: Growth factors induce nuclear translocation of MAP kinases (p42mapk and p44mapk) but not of their activator MAP kinase kinase (p45mapkk) in fibroblasts. J Cell Biol 122: 1079-1088, 1993.

31. Heinrich PC, Behrmann I, Müller-Newen G, Schaper F and Graeve L: Interleukin-6-type cytokine signalling through the gp130/Jak/STAT pathway. Biochem J 334: 297-314, 1998.

32. Mertens C, Zhong M, Krishnaraj R, Zou W, Chen X and Darnell JE Jr: Dephosphorylation of phosphotyrosine on STAT1 dimers requires extensive spatial reorientation of the monomers facilitated by the N-terminal domain. Genes Dev 20: 3372-3381, 2006.

33. Xu X, Sun YL and Hoey T: Cooperative DNA binding and sequence-selective recognition conferred by the STAT amino-terminal domain. Science 273: 794-797, 1996.

34. Bhat NR and Zhang P: Hydrogen peroxide activation of multiple mitogen-activated protein kinases in an oligodendrocyte cell line: Role of extracellular signal-regulated kinase in hydrogen peroxide-induced cell death. J Neurochem 72: 112-119, 1999.

35. Ishikawa $Y$ and Kitamura M: Anti-apoptotic effect of quercetin: Intervention in the JNK- and ERK-mediated apoptotic pathways. Kidney Int 58: 1078-1087, 2000.

36. Kim YK, Kim HJ, Kwon CH, Kim JH, Woo JS, Jung JS and Kim JM: Role of ERK activation in cisplatin-induced apoptosis in OK renal epithelial cells. J Appl Toxicol 25: 374-382, 2005.

37. Jo SK, Cho WY, Sung SA, Kim HK and Won NH: MEK inhibitor, U0126, attenuates cisplatin-induced renal injury by decreasing inflammation and apoptosis. Kidney Int 67: 458-466, 2005. 